\title{
Cubiertas históricas planas de tierra en el mediterráneo. Investigación para la caracterización del comportamiento hídrico de los materiales de las cubiertas de Ibiza
}

\author{
Historical flat roofs of earth in the Mediterranean. Research for the \\ characterization of the water behavior of the materials of the roofs of Ibiza
}

\begin{abstract}
M.E. Escudero Lafont ${ }^{(*)}$, S. García Morales ${ }^{(* *)}$
RESUMEN

Las cubiertas planas de tierra históricas se han descrito constructivamente por numerosos autores. Sin embargo, no se ha realizado un estudio científico de su comportamiento hídrico. Al ser una tipología extendida por los cinco continentes, este artículo se centra en las cubiertas de Ibiza y en las de las zonas que cultural e históricamente han tenido relación: España y África Bereber. Se compara y analiza el comportamiento de los materiales utilizados en la cubierta ante la lluvia, realizando ensayos de laboratorio para determinar la capacidad de infiltración y capacidad de campo de los materiales, especialmente de la última capa de la cubierta, caracterizando así cuantitativamente la impermeabilidad de las margas de Ibiza y explicando su buen comportamiento respecto a la lluvia.
\end{abstract}

Palabras clave: Terrado, capacidad de infiltración, cubierta de tierra, arquitectura vernácula.

\section{ABSTRACT}

The historic earth flat roofs have been described, in terms of construction, by numerous authors. However, a scientific study of its water behavior has not been carried out. Being a typology extended by the five continents, this article focuses on the earth flat roofs of Ibiza and those of the culturally and historically related areas: Spain and Berber Africa. It compares and analyzes the behavior of the materials used in the roof with respect to rainfall, performing laboratory tests to determine the infiltration capacity and water retention capacity of the materials, especially the top layer of the roof, quantitatively characterizing the impermeability of the marl from Ibiza and explaining its good behavior with respect to rain.

Keywords: Terrado, infiltration capacity, earth roof, vernacular architecture.

(*) Arquitecto. Escuela Técnica de Arquitectura de Madrid. Universidad Politécnica de Madrid (UPM) (España).

(**) Doctora Arquitecta. Departamento de Construcción. Escuela Técnica de Arquitectura de Madrid. Universidad

Politécnica de Madrid (UPM) (España).

Persona de contacto/Corresponding author: 10807escudero@gmail.com (M.E. Escudero).

ORCID: http://orcid.org/oooo-0oo2-7832-1432 (M.E. Escudero); http://orcid.org/oooo-ooo3-1106-1063 (S. García).

Cómo citar este artículo/Citation: Escudero-Lafont, M.E.; García-Morales, S. (2019). Cubiertas históricas planas de tierra en el mediterráneo. Investigación para la caracterización del comportamiento hídrico de los materiales de las cubiertas de Ibiza. Informes de la Construcción, 71(555): e301. https://doi.org/10.3989/ic.62786

Copyright: (C) 2019 CSIC. Este es un artículo de acceso abierto distribuido bajo los términos de la licencia de uso y distribución Creative Commons Reconocimiento 4.o Internacional (CC BY 4.0). 


\section{INTRODUCCIÓN}

Las cubiertas de tierra históricas se encuentran por gran parte de la geografía mundial, como arquitectura vernácula en viviendas, iglesias, ermitas o mezquitas, incluyendo la arquitectura militar.

La existencia de este tipo de cubierta está referenciada desde tiempos muy antiguos: en Egipto existen referencias arqueológicas (1) de su existencia en la Villa Deir al-Medina, 1500 a 1200 a.C. Geográficamente esta tipología se extiende por la cuenca del Mediterráneo, zonas de África y América, siempre en zonas climáticas secas, con fuertes diferencias térmicas y escasas lluvias anuales.

Los estudios realizados dentro del Proyecto Meda-Corpus ${ }^{1}$ en la cuenca mediterránea estiman que el 40\% de la arquitectura vernácula analizada responde a tipologías con cubiertas planas. En dicho Proyecto se describe, de un modo muy general, la sección básica de dichas cubiertas, las lesiones más comunes y las transformaciones sufridas en el último siglo.

La cubierta de tierra tradicional es una cubierta plana, con bajas pendientes (del 1 al 5\%) cuyo aislamiento e impermeabilización se realizan con capas de tierras arcillosas, mezcladas en ocasiones con otros materiales vegetales (Ver Figura 1). La mayoría de los autores convergen en esta definición de la cubierta como Gil Albarracín (2) Ortíz Moreno (3), Baglioni, (4), Verdera (5), Nourissier (6) o Sorreche Cuerva (7).

La tipología de cubierta de tierra ha sido estudiada y recogida por numerosos autores como Feduchi (8) o Flores (9), en sus tratados de arquitectura tradicional o vernácula, principalmente desde principios del siglo XX. Su localización, que se limita a ciertas provincias del litoral mediterráneo que le han ido dando una configuración particular a su arquitectura, ha despertado interés en los arquitectos de distintas épocas, que resaltan las diferentes respuestas constructivas encontradas en cada zona, como Blakstad (10) o Serra Rodríguez (11) en Ibiza, García Grinda (12) o Gíl Albarracín (2) en Almería, incluyendo croquis o dibujos de las mismas. Otros autores se limitan a describirla, sin apoyo gráfico como Más Hernández (13), Ortiz Moreno (3) o Sorroche Cuerva (7). Por último, al-

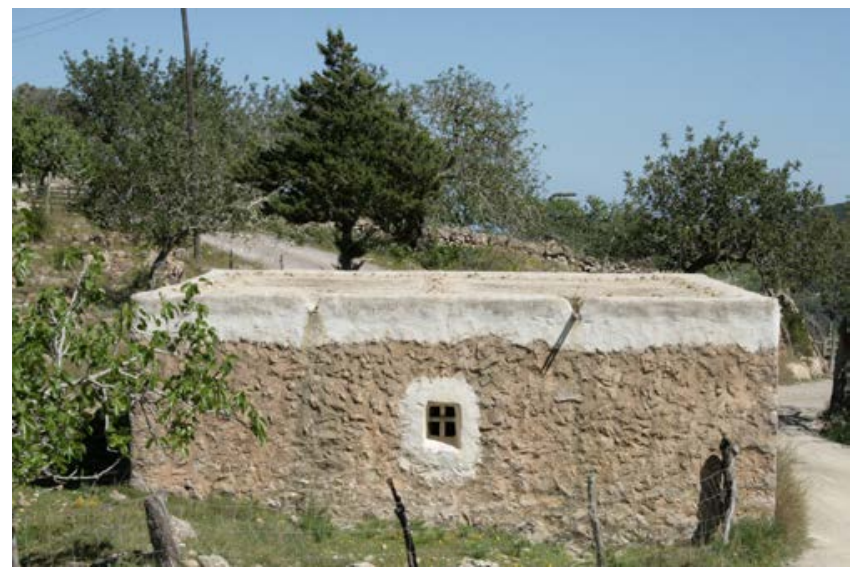

Figura 1. Molino de Sangre en Ibiza. gunos autores señalan sus características principales de protección ante el clima exterior como Eric Muhler (14) o López Osorio y Abadía (15).

Aquellos autores describen la forma constructiva de las cubiertas de tierra, identificando las distintas capas de materiales autóctonos. Muchos de ellos afirman el buen comportamiento de estas cubiertas ante las precipitaciones; pero a pesar de ese buen comportamiento justifican la sustitución de este sistema constructivo por técnicas modernas, debido a la necesidad de una conservación anual, frente al mantenimiento más dilatado en el tiempo de los materiales actuales.

La bibliografía en lo referente a las cubiertas, se limita a definir sus partes, materiales y espesores. Sin embargo, no se ha estudiado el comportamiento de los materiales ni las cualidades hídricas que les hacen supuestamente idóneos para su uso.

El presente trabajo forma parte de una Tesis en curso, cuyo objetivo es identificar y cuantificar el comportamiento hídrico de estas cubiertas planas de tierra y documentarlas, para evitar la pérdida del patrimonio cultural constructivo existente.

\section{DESCRIPCIÓN DE LA TIPOLOGÍA DE CUBIERTAS DE TIERRA}

Las cubiertas planas de tierra se realizan con los materiales presentes en el entorno de la construcción; aunque la sección tipo tiene algunas características comunes, lo cierto es que si se comparan diferentes ejemplos de zonas geográficas próximas entre sí, y con situaciones histórico-culturales similares, (península Ibérica, isla de Ibiza, África bereber), se aprecian variaciones de los materiales y de los espesores que las forman, aunque se mantienen siempre las mismas capas y su posición dentro de la sección de la cubierta.

Se relacionan siete cubiertas, (Figura 2) comparando materiales y espesores. Los datos previos se han obtenido de la bibliografía y de trabajo de campo.

Las tres primeras cubiertas son del África Bereber: las secciones definidas por Guibbert (16), de la zona de Al-Mizab (Figura 2A) (región del interior de Argelia, donde están las poblaciones de Garaïa, Daia Ben Dahoua y Berriane); otra de Djenné de Mali (Figura $2 \mathrm{C}$ ), zona inundable del río Niger, entre las ciudades Segou y Mopti, y la tercera sección africana ha sido descrito por Vicent Soriano (17) siendo la sección de los terrados marroquíes del oasis de Sukhüra (Figura 2B) (entre las cordilleras de Alto Atlas y del Saghro y entre las ciudades de Uarzazate y Boulmalne Dades en el valle del río "Dades").

La siguiente cubierta estudiada (Figura 2D) es de la isla de Ibiza, corresponde al molino de sangre de la Figura 1. Está formada por tres capas sobre la estructura de madera: una de $5 \mathrm{~cm}$ de algas secas (posidonia), una capa intermedia de tierra de carbonera (material residual en la producción de carbón) de $10 \mathrm{~cm}$ y la capa superior formada por $10 \mathrm{~cm} \mathrm{de}$ margas, denominadas "argilas" (que significa arcilla).

\footnotetext{
${ }^{1}$ MEDA, EUROMED HERITAGE. Proyecto euro-mediterráneo, realizado durante los años 1998-2001, cuyo propósito es el estudio y catalogación de las tipologías constructivas del patrimonio no monumental de la cuenca mediterránea. De él surgió CORPUS como observatorio de la arquitectura tradicional mediterránea. (www.meda-corpus.net)
} 


\begin{tabular}{|c|c|c|c|}
\hline 48 & $2-x$ & & $10 \mathrm{~cm}$ \\
\hline$\therefore \therefore \quad$ & $2 x$ & $3 \mathrm{sg} / \mathrm{x}$ & 10 \\
\hline$\therefore \cdots \cdots$ & $\alpha \%$ & $\therefore \ldots$ & 10 \\
\hline$\therefore \quad 1$ & $k^{2}-1$ & 61 & 10 \\
\hline
\end{tabular}

$2 \mathrm{~A}$

$2 B$

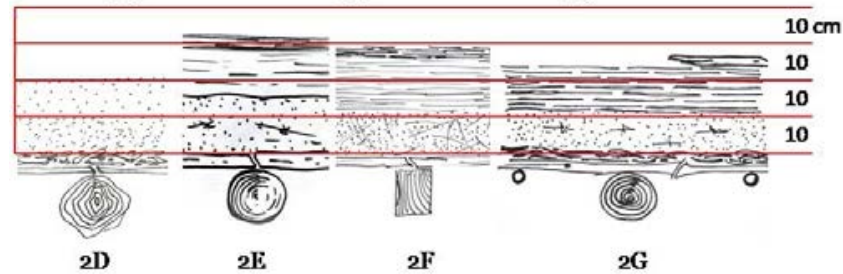

Figura 2. Representación de las secciones de cubiertas planas de tierra.

Por último se analizan tres cubiertas en la península ibérica, de la zona de Granada y Almería. Se corresponden con las secciones descritas por Gil Albarracín para la provincia de Almería (Figura 2G), de Ortiz Moreno para La Alpujarra de Granada (Figura 2F) y la descripción para los terrados del Marquesado de Zenete en Granada por Sorroche Cuerva (Figura 2E).

En el diagrama (Figura 3) se muestran las capas de las $7 \mathrm{cu}-$ biertas, con sus espesores y naturaleza. En esta comparativa podemos observar que aunque las secciones siguen unos criterios unitarios, el uso de los materiales de la zona implica variaciones en los espesores de las capas y de la cubierta.

La falta de publicaciones sobre el comportamiento hídrico de estas cubiertas, ni de la respuesta de la sección ante las precipitaciones, obliga a realizar unas primeras hipótesis sobre dicho comportamiento, especialmente en lo que se refiere a la capa superior, cuya misión, y en conformidad a la bibliografía, sería la impermeabilización de la cubierta. Posteriormente se ha buscado confirmar dicha hipótesis mediante unos primeros ensayos. En la bibliografía se identifica la capa vegetal inferior como capa de protección de humedad de la estructura de madera o como aislamiento térmico. A la capa intermedia no se le asigna una función específica. Podría responder a una razón de aislamiento térmico, pero eso no explicaría las variaciones de espesor de dicha capa de unos ejemplos a otros. Si lo que determina el espesor de la capa intermedia es la necesidad de absorber el agua que se infiltra tras la escorrentía, cuanto más estrecha la capa, indicaría menor infiltración a través de la capa exterior.
En el caso de las cubiertas de España se tiene tres secciones, cuya capa de impermeabilización es arcilla magnésica; esta capa superior varía de 2,5 a $10 \mathrm{~cm}$, y la capa intermedia en dos de los casos, es del mismo material que la capa superior.

Si se considera como hipótesis de partida que las siete cubiertas consiguen la misma efectividad ante las precipitaciones, la sección de espesor menor sería la más eficiente de ellas.

El papel de la capa exterior ante la precipitación debería ser conducir la escorrentía del agua hacia la gárgola, y reducir la infiltración. Si esta capa se deteriora, ya sea por las limitaciones del material o por los cambios físicos de la superficie por efectos externos (fisuras por cambios térmicos o de humedad, plantas, etcétera), se podría producir una infiltración de agua a través de ella, llegando a la capa intermedia, la cual debe retener ese agua infiltrada y evitar que alcance la capa interior.

Como ya queda dicho, el mayor espesor de esta capa intermedia indicaría o el temor al agua infiltrada o la necesidad de un mayor aislamiento térmico.

La Figura 3 muestra que las cubiertas de Ibiza tiene un espesor total claramente inferior a las africanas y entre las demás cubiertas españolas. La capa de acabado formada por las margas tiene un espesor medio de $10 \mathrm{~cm}$ y no se producen incrementos de los espesores a lo largo del tiempo por refuerzo o reparación. La capa intermedia se mueve en el entorno de los $10 \mathrm{~cm}$. Esto podría significar una mayor eficiencia de esta cubierta.

Para comprobar esta hipótesis de la mayor eficiencia de las margas es necesario ensayar el comportamiento de los materiales de las capas de remate superior, e identificar sus características propias respecto a la lluvia.

\section{OBJETIVO}

El objetivo del presente artículo es mostrar el comportamiento ensayado de las margas utilizadas en la cubierta tradicional de Ibiza, para comprobar si la menor sección de esta cubierta se debe al mejor comportamiento del material por sus propiedades hídricas.

Para tener elementos de comparación se han ensayado también otros materiales: una arcilla (local, de origen granítico, aluvial) y una arena de miga (material más permeable y con un comportamiento previsiblemente no adecuado para cubierta), determinando para los tres casos los siguientes valores: capacidad de infiltración y capacidad de campo (saturación).

\begin{tabular}{|c|c|c|c|c|c|c|c|}
\hline \multirow{2}{*}{$\begin{array}{l}35 \mathrm{~cm} \\
30 \mathrm{~cm}\end{array}$} & \multirow[t]{2}{*}{$\begin{array}{c}\text { tierra } \\
\text { compostada }\end{array}$} & & & & \multirow[b]{2}{*}{$\begin{array}{l}\text { Launa } \\
\text { apretada }\end{array}$} & \multirow[b]{2}{*}{$\begin{array}{l}\text { Launa } \\
\text { cernida }\end{array}$} & \\
\hline & & Barro y paja & Lodo y paja & & & & \\
\hline $25 \mathrm{~cm}$ & & & & & Launa, & Launa, & Roya, \\
\hline $20 \mathrm{~cm}$ & Tierra & & Tierra & Margas & arcilla & arcilla & arcilla \\
\hline $15 \mathrm{~cm}$ & apisonada & Tierra & Orgánica & & Magnésica & Magnésica & Magnésica \\
\hline $10 \mathrm{~cm}$ & & & & Tierra & Tierra y & Tierra y & Barro, \\
\hline \multirow[t]{3}{*}{$5 \mathrm{~cm}$} & & & & Carbonera & paja & paja & yeso o cal \\
\hline & & Adobe & Telas viejas & Posidonia & \multirow[b]{2}{*}{$\begin{array}{l}\text { M. Zenete } \\
\quad(2 \mathrm{E})\end{array}$} & \multirow[b]{2}{*}{$\begin{array}{c}\text { Alpujarras } \\
(2 \mathrm{~F})\end{array}$} & Adelfa \\
\hline & $\begin{array}{c}\text { Al-Mizab } \\
(2 \mathrm{~A})\end{array}$ & $\begin{array}{c}\text { Sukhüra } \\
(2 \mathrm{~B})\end{array}$ & $\begin{array}{c}\text { Djenné } \\
(2 \mathrm{C})\end{array}$ & $\begin{array}{l}\text { Ibiza } \\
(2 \mathrm{D})\end{array}$ & & & $\begin{array}{c}\text { Almería } \\
(2 \mathrm{G})\end{array}$ \\
\hline
\end{tabular}

Figura 3. Comparativa de secciones de cubierta. 


\subsection{Métodos actuales de cálculo de la infiltración}

El comportamiento de las aguas en el terreno esta profusamente estudiado por los ingenieros de caminos y agrónomos. Para la caracterización del comportamiento de la cubierta de tierra, se trabaja con la parte de la hidrología que estudia las propiedades del terreno superficial.

En los tratados clásicos de hidrología se analiza el comportamiento del terreno para determinar el potencial de acuíferos de la zona y su explotación. Los conceptos con los que se trabaja son, principalmente, la capacidad y velocidad de infiltración.

Las condiciones en las que se trabaja en una cubierta plana de tierra, con un espesor reducido, limitan el movimiento del agua a la infiltración: en descenso por capilaridad y gravedad, o en ascenso por evaporación y capilaridad, no teniendo gran influencia en su comportamiento el gradiente hidráulico.

El concepto que determina el comportamiento de nuestra capa superior es la capacidad de infiltración, ya que es la que determina la presencia y cuantía de fluido en capas inferiores.

La Infiltración o humedecimiento del suelo es el volumen de agua que procedente de las precipitaciones, en un determinado tiempo, atraviesa la superficie del terreno y ocupa total o parcialmente los poros del suelo (18). La infiltración depende de las características de la tierra y del fluido: compactación del terreno, la presencia de vegetación o la pendiente de la superficie, la humedad previa del terreno, su temperatura, el espesor de la lámina de agua, su turbidez y la temperatura de la misma.

Para determinar la capacidad de infiltración los ingenieros utilizan dos tipos de infiltrómetros: los de tipo "inundador" (Müntz) o de cilindros concéntricos, y los de cilindro excavado (Porchet). Se utilizan también otros métodos de simulación de lluvia en parcelas más amplias. (18)

De acuerdo con la teoría, el análisis del comportamiento del agua de lluvia que llega a la superficie de la tierra (agua precipitada, P) (Figura 4), determina que una parte se infiltra hacia el interior (agua infiltrada, I) y otra parte corre por la superficie (agua de escorrentía, E). Del agua infiltrada en el terreno, una parte se retiene entre los poros del terreno, según la Capacidad de Campo (CC) del mismo, y otra parte desciende a capas inferiores (agua gravídica, G).

El agua de las precipitaciones $(\mathbf{P})$ satura los huecos del terreno y desciende por gravedad, éste agua gravídica (G), agua que no retiene el terreno, sería la que podría producir la humedad en las cubiertas de tierra, y por lo tanto, sería éste agua la que debería ser retenida en la capa intermedia de tierra se- gún su propia capacidad de campo. El agua capilar continua o sostenida sería el agua de la zona saturada del terreno.

$$
\begin{gathered}
P=E+I \\
I=C C+G+E_{v}
\end{gathered}
$$

Por tanto, la Capacidad de Campo [3] se define como el grado de humedad de una muestra del terreno tras perder el agua gravídica; se relaciona con la Capacidad de Retención Específica (ms) [4] según las siguientes fórmulas:

$$
\begin{gathered}
C C=100 \times\left[\left(P_{f}-P_{i}\right) / P_{i}\right] \\
m_{s}=V_{s} / V_{t} \\
m_{s}=C C \times \delta_{t}
\end{gathered}
$$

Siendo $V_{s}$ el volumen de agua infiltrada una vez evacuada el agua gravídica, $V_{t}$ el volumen total del terreno y $\delta_{t}$ la densidad aparente del terreno. Para un adecuado comportamiento de la cubierta de tierra, se requeriría una capacidad de campo de la capa intermedia equivalente al agua gravídica filtrada por la capa exterior, para el caso de la precipitación máxima de la zona climática.

\section{MATERIAL Y MÉTODOS}

\subsection{Hipótesis de trabajo y teorías para el cálculo}

En una cubierta de tierra plana, la mayor parte del agua precipitada (P) se evacúa mediante escorrentía (E), por las gárgolas. Como una parte se infiltra (I), la cubierta funcionará bien si se cumplen las condiciones que hemos descrito más arriba. Por ello es tan importante conocer la infiltración $(\boldsymbol{f})$ y la capacidad de campo (CC) de las capas exterior e intermedia.

En el presente trabajo se ha adaptado el método del infiltrómetro de Müntz al laboratorio (Figura 5). Si se compara con la figura 4, se ve que el agua de escorrentía (E) se ha transformado en agua retenida (R). En el primer momento del ensayo se mantiene la presión constante de agua sobre la superficie al ir incorporando agua cada 5 minutos y conservando el nivel de $\mathbf{R}$. Posteriormente se deja de incorporar agua y todo $\mathbf{R}$ termina infiltrándose a través de la tierra. Esto lleva a la saturación total de la sección del material, y a identificar el comportamiento en una sección limitada de tierra.

Se limitan las pérdidas por evaporación directa $\left(\mathbf{E}_{\mathbf{v}}\right) \operatorname{de} \mathbf{R}$ y $\mathbf{G}$, tapando la probeta y reduciendo la superficie de pérdida. Por ello, el tiempo de retención del agua es inverso a la capacidad de infiltración, una vez saturado el terreno.

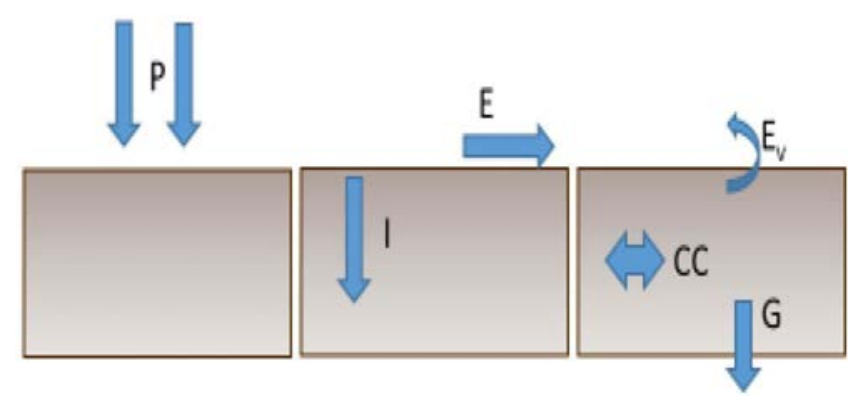

Figura 4. Recorrido del Agua en tierra [1], [2], [3], [4], [5].

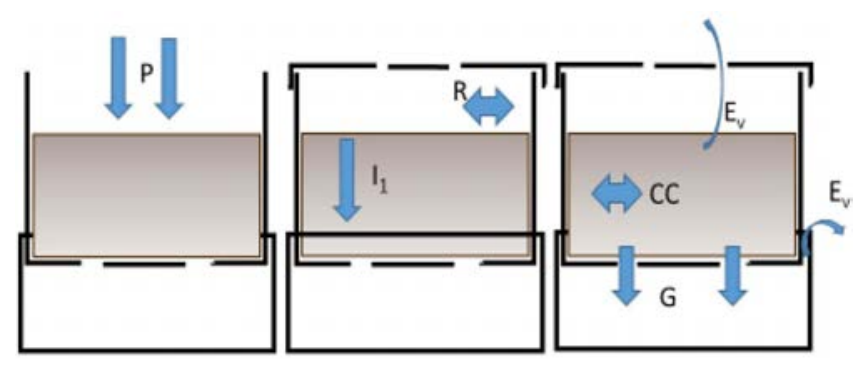

Figura 5. Recorrido del agua en probeta [6], [7]. 


$$
\begin{gathered}
P=R+I_{1} \\
I_{1}=C C+G+E_{v}
\end{gathered}
$$

En el ensayo de estas probetas, se espera a que todo $\mathrm{P}$ pase por la sección, analizando así el comportamiento de esta ante una solicitación límite. Al anular la pendiente y la escorrentía se fuerza la infiltración.

\subsection{Materiales para el ensayo}

- MARGAS: se toman muestras de dos distintas "Covas", (canteras locales de Ibiza):

- Cova de Can Miquelet (Sant Carles de Peralta) (Marga): 3\% Calcita, 50\% Dolomita, 47\% Filosilicatos de los cuales: $98 \%$ Illita-mica y $2 \%$ Clorita.

- Cova en PM-803 Sant Josep (Margo caliza): 10\% Cuarzo, 70\% Calcita, 1\% Dolomita, 1\% Pirita, 18\% Filosilicatos de los cuales: $75 \%$ Esmectita, 24\% Illita - mica y 1\% Caolinita.

- Tierra de Madrid, Franco Arcillo Arenosa, SW, arena bien graduada con pocos finos (26\%) cuya caracterización mineralógica es: cuarzo, feldespato potásico, filosilicatos y plagioclasas. Formados los filosilicatos por aproximadamente un $40 \%$ Esmectita, un $40 \%$ de illita - mica y un 20\% de caolinita. Extraídas a 28km de la Ciudad, en la zona de encuentro entre las riberas del río Jarama y Guadalix, cribada por tamiz de $125 \mathrm{~mm}$.

- ARENA DE MIGA: (granulometría 97,8\% de 4 a >0,062) de cantera, para tener un valor comparativo de un material de alta capacidad de infiltración.

\subsection{Diseño de las probetas}

La probeta se realiza con un tubo cilíndrico transparente de diámetro $900 \mathrm{~mm}$ e interior de $845 \mathrm{~mm}$, para poder ver el descenso del agua por la sección de la misma. La base se realiza con geotextil fijado al perímetro, que impide la pérdida de materia y permite la salida de G. Está provista de tapa con dos pequeñas aberturas para equilibrar la presión atmosférica, y por tanto permitir la infiltración de R, pero evitando la evaporación (Figura 6).

La probeta se apoya sobre una rejilla que la separa del depósito de $\mathbf{G}$.

Este depósito es de diámetro $1000 \mathrm{~mm}, 1 \mathrm{~cm}$ mayor a la probeta con el mismo fin de reducir $\mathbf{E}_{\mathbf{v}}$.

Se rellena la probeta con la correspondiente tierra en tongadas de $2,5 \mathrm{~cm}$ de espesor, y compactadas con un peso equivalente a $80,6 \mathrm{~g} / \mathrm{cm}^{2}$.

\section{DESCRIPCIÓN DEL ENSAYO}

\subsection{Determinación de la cuantía de agua}

Se analizan los datos climáticos locales de Ibiza, obtenidos de la Agencia Estatal de Meteorología de España, AEMET, identificando la cuantía a la que ha de responder la cubierta. Se consideran las precipitaciones medias mensuales y los valores extremos de máximas precipitaciones desde 1944 .
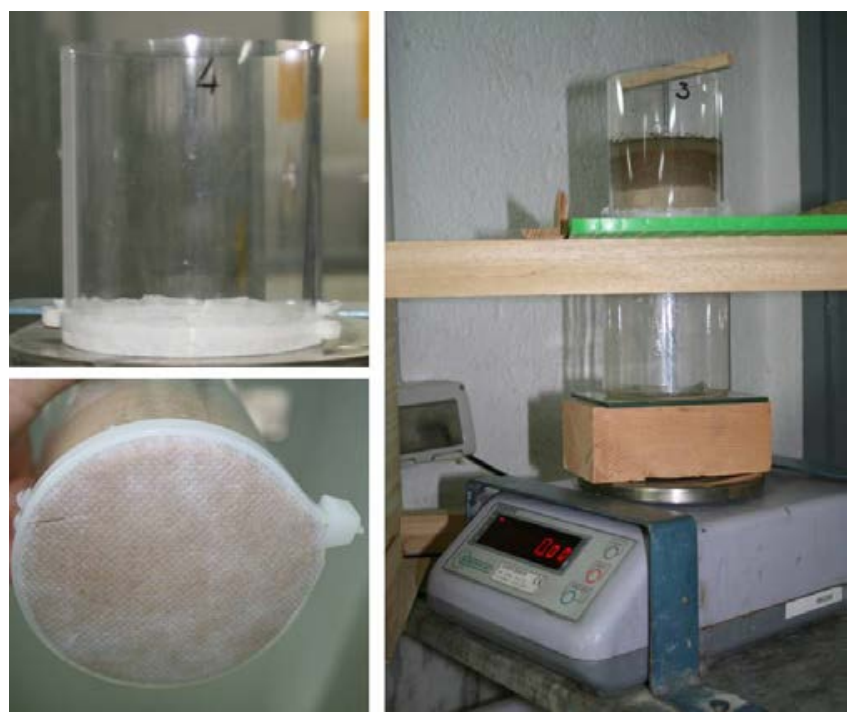

Figura 6. Probeta, base de geotextil para la retención de la tierra con libre paso del agua y ensayo.

El agua $\mathbf{P}$ sobre las probetas será la equivalencia a la media mensual $\left(29 \mathrm{~L} / \mathrm{m}^{2}\right)$ es decir, 162,63 gr pero mantenida, la intensidad durante una hora y sin escorrentía, por lo que el equivalente de lluvia es superior.

\subsection{Fases del ensayo}

\subsubsection{Fase Primera}

Se vierte $\mathbf{P}$ y cada 5 minutos se va reponiendo la cantidad necesaria para mantener el nivel. La cuantía de agua repuesta, equivale a la que se ha infiltrado en dicho tiempo, y con ello se cuantifica la Capacidad de Infiltración de la tierra analizada, y su evolución [8].

$$
f=\frac{\Delta W}{A}
$$

\subsubsection{Fase Segunda}

Transcurrida la hora en la que se mantiene el nivel de $\mathbf{R}$, se deja de reponer ésta. Se va midiendo, a partir de ese momento, el ritmo de infiltración, y se va pesando periódicamente $\mathbf{G}$.

Los datos que se analizan son el comportamiento del material ante la infiltración de agua, retención en superficie y su saturación. Estos datos permitirán determinar el comportamiento de dicho material ante las precipitaciones.

Con la ayuda de una balanza de precisión, determinamos las cuantías de $\mathbf{P}$ y G. Determinando los ritmos en el desplazamiento del agua.

Así mismo medimos las alturas de $\mathbf{R}$ y la profundidad de $\mathbf{I}$.

Con los resultados se obtienen $\mathbf{C C}$ de los distintos terrenos, su capacidad de retención, y con ello la sección necesaria para una determinada cuantía de precipitación.

Los ensayos se realizaron durante el mes de julio y con agua a temperatura ambiente. 


\section{RESULTADOS}

Con los resultados se obtienen unas gráficas que permiten observar el ritmo de infiltración, el momento de la aparición de $\mathbf{G}$ y el grado de saturación de la tierra.

En las arenas de miga (Figuras 7 y 8), G hace su aparición entre los 5 y 15 minutos del comienzo del ensayo (línea roja). Tras la hora de mantenimiento de $\mathbf{R}$, la infiltración de esta es completa una hora después (línea azul) y 15 minutos después, deja de caer G. El valor de $\mathbf{C C}$ se obtiene entonces [1] dando valores de 25,04 y 24,30\%. La arena de miga está saturada prácticamente desde el comienzo del ensayo (los valores de I se mantienen constantes).

Los datos correspondientes a las arcillas (Figuras 9 y 10) son: $\mathbf{G}$ hace su aparición entre los 15 y 20 minutos del comienzo del ensayo. Pasada la hora de $\mathbf{R}$ constante, se infiltra en su totalidad entre dos horas y 20 minutos y cuatro horas 55 minutos desde dicho momento. La estabilidad de I en la tierra, se obtiene a tres horas 20 minutos y cuatro horas 40 minutos de la totalidad del tiempo con unos valores de $\mathbf{C C}$ de 23,40 y $22,80 \%$.

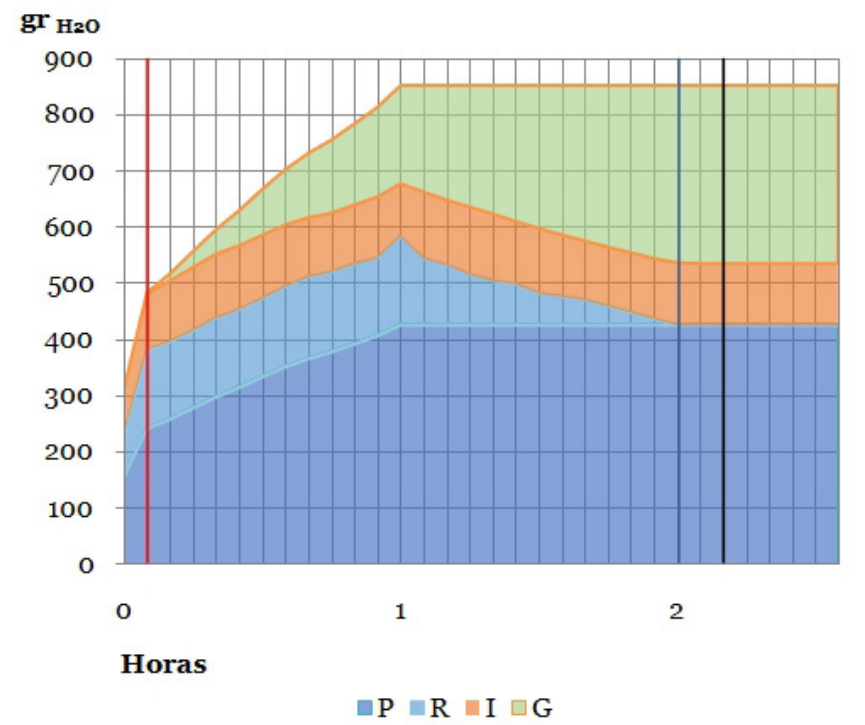

Figura 7. Ensayo fase 1 y 2 con Arena de miga probeta 1.
En el caso de las margas, el comportamiento mejora con respecto a las tierras anteriores:

En la probeta de la marga 1 (Figura 11), se retrasa la aparición de $\mathbf{G}$ hasta tres horas y diez minutos después del comienzo del ensayo. Siendo necesarias 78 horas para que se infiltre todo $\mathbf{R}$. Cinco horas antes ya no aparece $\mathbf{G}$ y llega a $\mathbf{C C}$ estable de valor 40,50\%, 1 hora y 45 minutos antes de la infiltración total de $\mathbf{R}$.

En el caso de la segunda marga (Figura 12), tras 49 horas, no se produce $\mathbf{G}$, y alcanza estabilidad en su $\mathbf{C C}$ de $22,01 \%$ a las 23 horas, manteniendo $21 \mathrm{~mm}$ de $\mathbf{R}$.

Se comprueba el buen comportamiento de las margas, que prácticamente no dejan pasar agua gravídica.

A continuación se presenta un gráfico que expresa la velocidad con que aparece el agua gravídica (Figura 13). Las líneas son rectas (velocidad constante) hasta alcanzar la $\mathbf{C C}$ de cada material. Una menor pendiente indica un mejor comportamiento de la probeta. La velocidad en el caso de la arena de miga es $24,96 \mathrm{~L} / \mathrm{m}^{2} \mathrm{~h}$ y 25,48 , de 7,59 y $7,37 \mathrm{~L} / \mathrm{m}^{2} \mathrm{~h}$

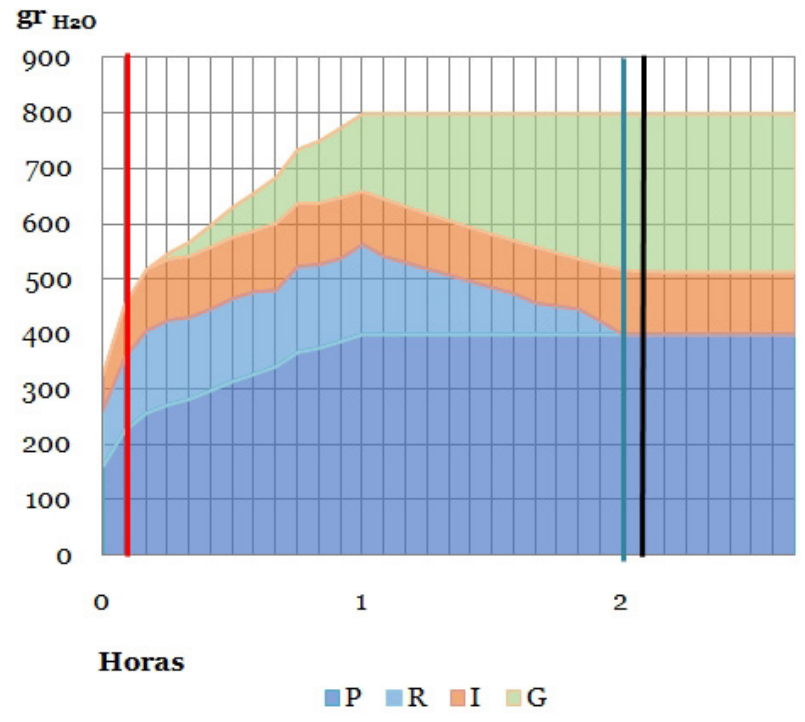

Figura 8. Ensayo fase 1 y 2 con Arena de miga probeta 2.

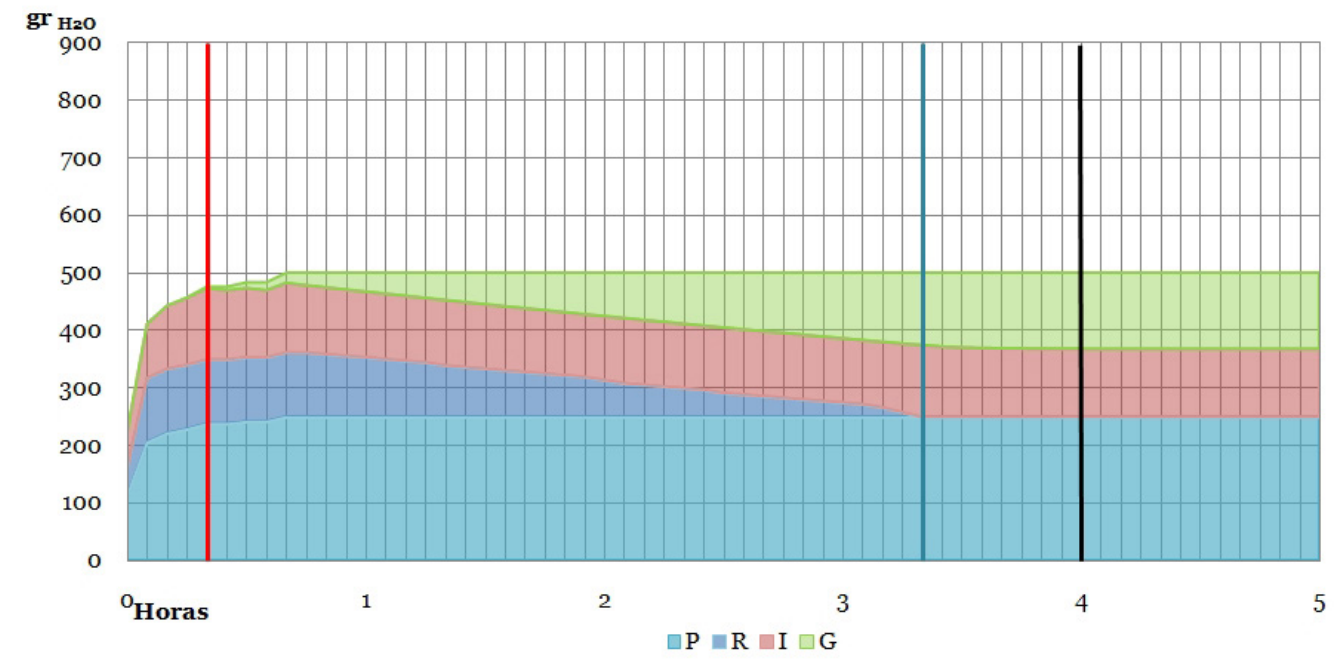

Figura 9. Ensayo fases 1 y 2. Arena Arcillosa de Madrid Probeta 1. 


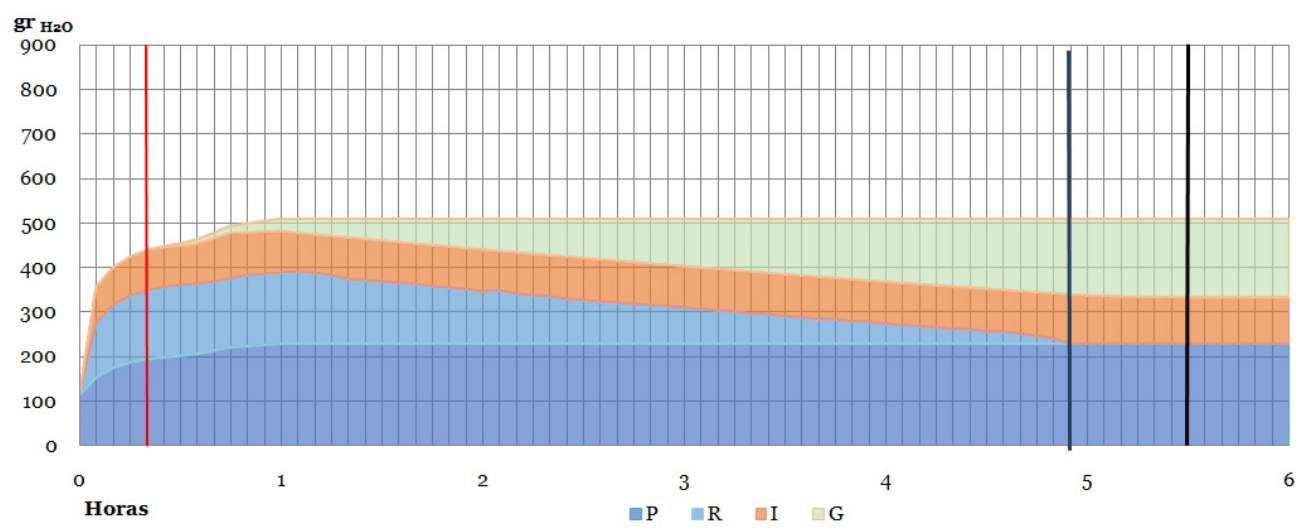

Figura 10. Ensayo fases 1 y 2. Arena Arcillosa de Madrid Probeta 2.

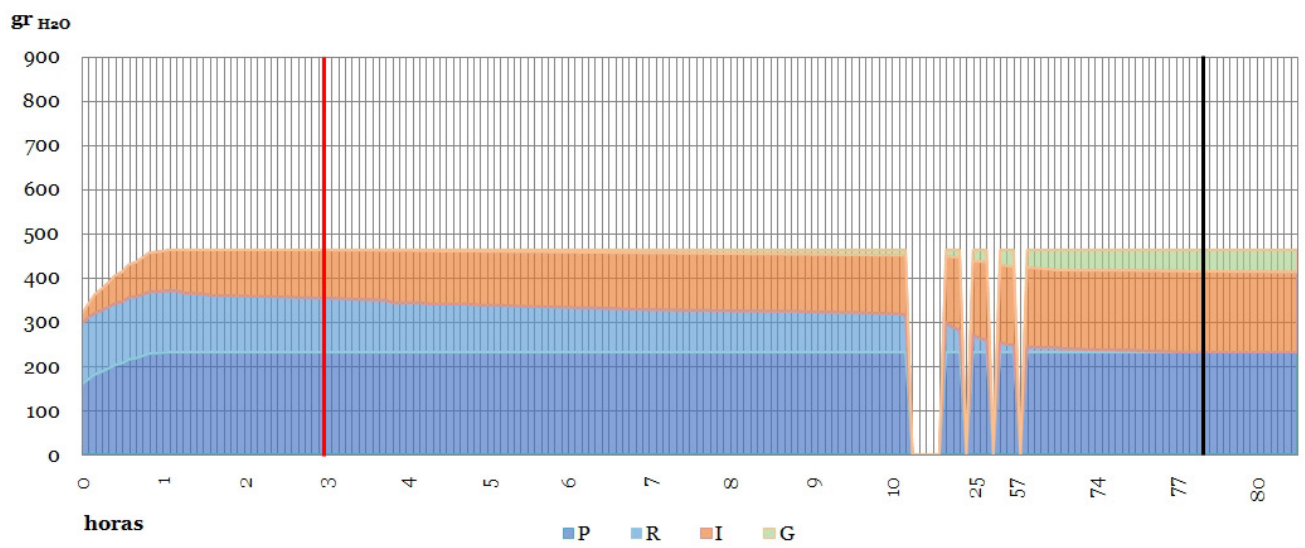

Figura 11. Ensayo fases 1 y 2. Marca de Can Miquelet, Ibiza.

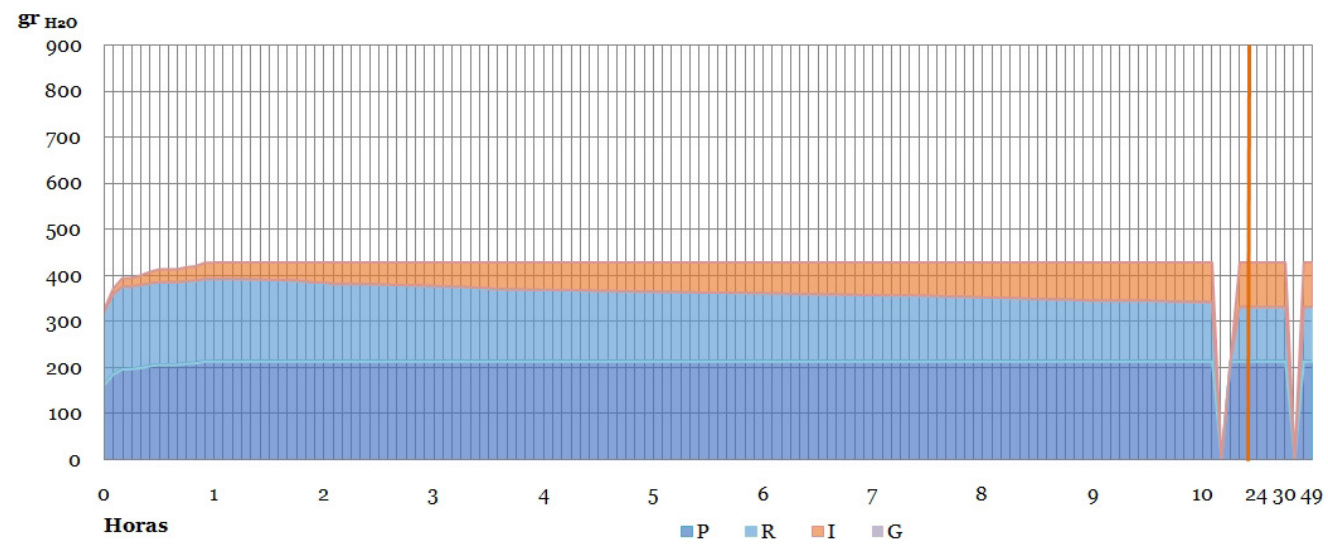

Figura 12. Ensayo fases 1 y 2. Margo Caliza de Ctra. PM-803 Sant Josep, Ibiza.

para la arena arcillosa y de 0,28 y $0,0 \mathrm{~L} / \mathrm{m}^{2} \mathrm{~h}$ en las margas de Ibiza.

Respecto a la capacidad de infiltración ( $\boldsymbol{f}$ ) de las muestras (Figura 14), en todos los casos, $\boldsymbol{f}_{\boldsymbol{i}}$ es mayor que los datos sucesivos. En el caso de las arenas de miga el valor (11 y $15 \mathrm{~L} / 5$ $\mathrm{min} \cdot \mathrm{m}^{2}$ ) es muy superior a los posteriores, que se mantienen en el entorno de $3,5 \mathrm{~L} / 5 \mathrm{~min} \cdot \mathrm{m}^{2}$ de forma ondulante y aparentemente constante. Si la arena de miga forma casi una línea con un punto de inflexión para mantenerse horizontal, en el caso de las arcillas, la línea que forman los valores obtenidos es una curva con tendencia a reducir $\boldsymbol{f}$.

Las margas no tienen una diferencia de valores tan marcada, pero claramente la capacidad desciende, siendo menor que en arenas y arcillas (Tabla 1).

Los valores promedios a partir de los 10 minutos son de 2,98 y $2,86 \mathrm{~L} / \mathrm{m}^{2}$ para las arenas, $0,72 \mathrm{y} 1,24 \mathrm{~L} / \mathrm{m}^{2}$ para las arcillas y $0,84 \mathrm{~L} / \mathrm{m}^{2}$ para la marga 1 y $0,40 \mathrm{~L} / \mathrm{m}^{2}$ para la marga 2 . 


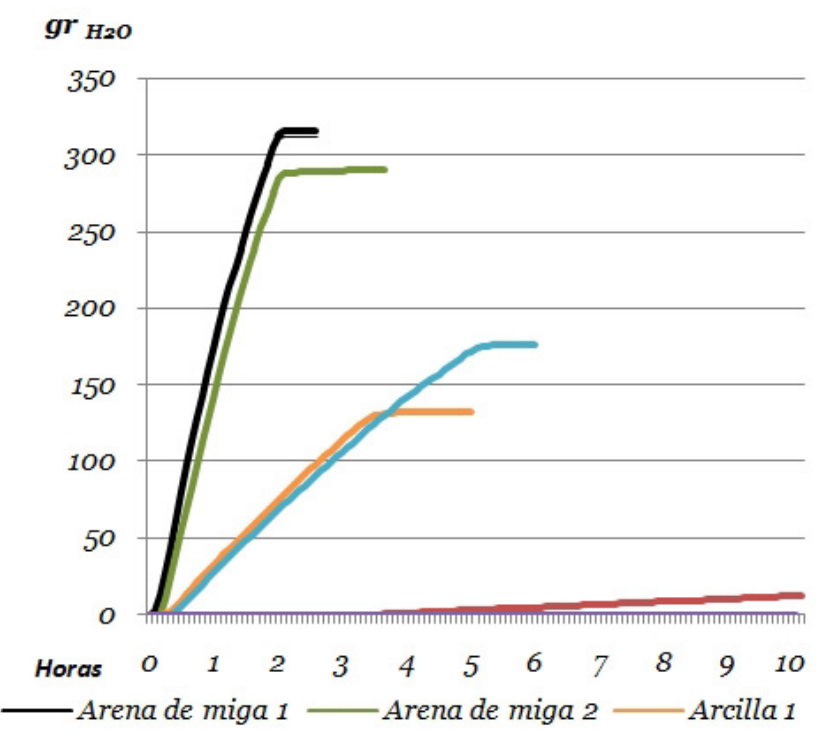

- Arcilla $2 \quad$ Margas $1 \quad$ Margas 2

Figura 13. Gráfica de Agua gravídica.

Tabla 1. Valores de los materiales de estudio

\begin{tabular}{|c|c|c|c|c|c|c|}
\hline \multirow{3}{*}{ Material } & $f_{\mathrm{i}}$ & $f_{\mathrm{c}}$ & $\mathrm{CC}$ & $V_{G}$ & G & $G: \Delta t\left(t_{i} ; t_{f}\right)$ \\
\hline & $\mathrm{L}$ & $\mathrm{L}$ & \multirow{2}{*}{$\%$} & $\mathrm{~L}$ & \multirow{2}{*}{$\%$} & \multirow{2}{*}{$\mathrm{h} ; \mathrm{h}$} \\
\hline & $\mathrm{m}^{2} \mathrm{mn}$ & $\mathrm{m}^{2} \cdot \mathrm{mn}$ & & $\mathrm{m}^{2} \cdot \mathrm{h}$ & & \\
\hline A.M. (P1) & 2,20 & 0,60 & 25,04 & 24,96 & 74,16 & $00: 05 ; 02: 15$ \\
\hline A.M. (p2) & 3,00 & 0,56 & 24,30 & 25,48 & 72,54 & $00: 15 ; 02: 05$ \\
\hline A. A. (p1) & 3,34 & 0,14 & 23,40 & 7,59 & 52,87 & $00: 15 ; 04: 00$ \\
\hline A. A. (p2) & 1,45 & 0,25 & 22,80 & 7,37 & 62,85 & $00: 20 ; 05: 25$ \\
\hline Marga 1 & 0,44 & 0,17 & 40,50 & 0,28 & 21,46 & $03: 10 ; 73: 50$ \\
\hline Marga 2 & 0,83 & 0,08 & 22,01 & 0,00 & 0,00 & 00:00 ; 00:00 \\
\hline
\end{tabular}

\section{CONCLUSIONES}

Como resultado de este trabajo se puede concluir que las margas de Ibiza han demostrado ser materiales de impermeabilización excelentes, incluso comparadas con las arcillas, puesto que el agua gravídica que atraviesa la capa de material, el "gotear" (G) es muy baja, y porque se retrasa esta como mínimo hasta las tres horas, y eso en el caso extremo que se ha simulado en los ensayos, que equivaldría a una lluvia de $30 \mathrm{l} / \mathrm{m} 2$ mantenida en un tiempo superior a 10 horas. El mejor material ha resultado ser la Margo Caliza de Ctra. PM-803 Sant Josep. En el ensayo realizado, la velocidad de goteo en el caso de la arena de miga es 24,96 y $25,48 \mathrm{~L} / \mathrm{m}^{2} \mathrm{~h}$, de 7,59 y $7,37 \mathrm{~L} / \mathrm{m}^{2} \mathrm{~h}$ para la arcilla, y de 0,28 y o,o L/ $/ \mathrm{m}^{2} \mathrm{~h}$ en las margas de Ibiza.

En las arcillas, toda el agua termina infiltrándose a las 3-5 horas, y parte de ella (alrededor del $23 \%$ ) queda reteni-

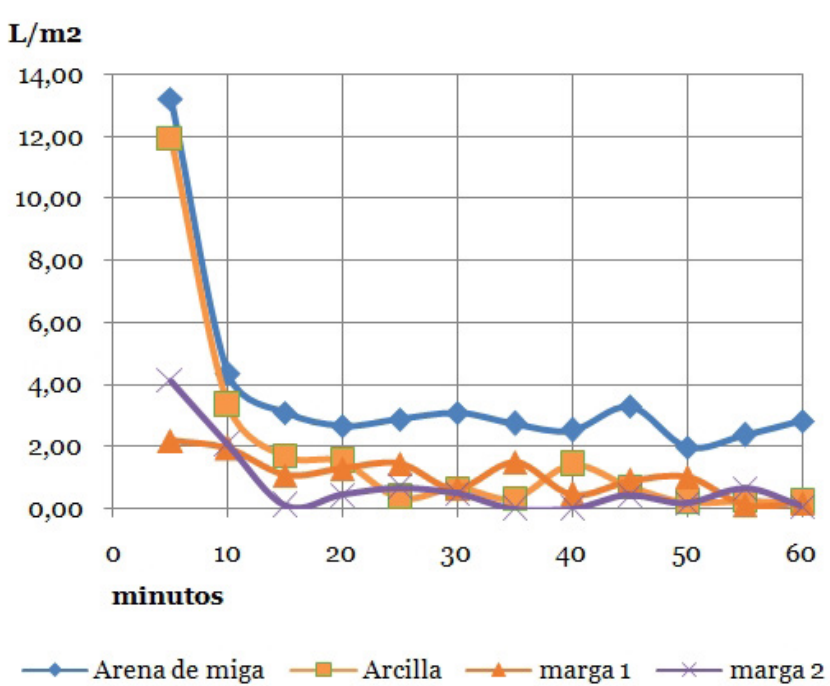

Figura 14. Gráfica de Capacidad de Infiltración de los materiales (f).

da (CC). Un 53-63\% del agua llegaría a atravesar la capa de $5 \mathrm{~cm}$, y sería capaz de gotear hacia la capa intermedia en una cubierta de tierra. Es decir, el punto crítico en una cubierta cuya capa superior estuviera ejecutada con estos materiales sería la capa intermedia, que tendría que ser capaz de absorber ese 53-63\% de agua, sin dejarla gotear al interior. Las soluciones constructivas que hemos podido documentar manejan espesores de esta capa entre 12 y 30 $\mathrm{cm}$, dependiendo posiblemente de la impermeabilidad del material.

En las margas, el agua superficial (R) se retiene mucho tiempo (más de 48 horas) El agua infiltrada absorbida (CC) en la capa exterior varía: entre el 22 y el $40 \%$. Muy poca agua $(1,3 \%)$ llegaría a gotear $(\mathbf{G})$ a las capas intermedias en las 5 primeras horas, esta capa tendría que haber retenido $4,45 \mathrm{~L} / \mathrm{m}^{2}$. Si esta tierra intermedia hubiese sido de arena de miga, con solo un espesor de $5 \mathrm{~cm}$, lo hubiera retenido sobradamente, siendo una quinta parte de su CC. Al final del ensayo, el agua gravídica alcanza el $46 \%$ de la CC de la arena de miga con el mismo espesor. Es decir, la cubierta ibicenca con esta marga y una capa intermedia de $5 \mathrm{~cm}$ de arena, resultaría impermeable. En la Marga 2, incluso, trascurridas 48 horas, no llega a producirse goteo. Parece que esto podría explicar el por qué los espesores de las cubiertas de Ibiza son inferiores a las otras cubiertas de tierra y que en el mantenimiento de estas cubiertas no se incrementan espesores, manteniéndose constante a lo largo de su vida útil.

Este ensayo previo a la actuación en las cubiertas de tierra planas, tanto para su reparación, rehabilitación o de nueva ejecución, permitiría determinar la sección mínima necesaria para la tierra local o la composición de acabado final.

\section{REFERENCIAS}

(1) Solé, G., Calzada, J., Castelreanas, A. (1988). Los grandes descubrimientos de la Arqueología. Vol. II, p. 155-163, Barcelona: Planeta - De Agostini.

(2) Gil Albarracín, A. (1992). Arquitectura y Tecnología Popular en Almería, p. 105 -142, Almería: Griselda Bonet Girabet GBG.

(3) Ortiz Moreno, E. (2005). Utopía. Márgenes. Apuntes de Arquitectura en la Alpujarra (Tesis Doctoral no publicada). Madrid: ETSAM. 
(4) Baglioni, E. (2011 septiembre). Il Patrimonio Architettonico in Terra Cruda. En Congreso de Arquitectura de Tierra en Cuenca de Campos (pp. 77-88), Valladolid: Cátedra Juan de Villanueva ETSAV.

(5) Verdera, C., Lenaerts, C. (2002). Detalles de la Arquitectura Rural Ibicenca. En Arquitectura y Espacio Rural en Ibiza (pp. 113-123), Illes Balears: Eivissa, COOA.

(6) Noussier, G., Reguant, J., Casanovas, J., Graz, C. (2002). Arquitectura Tradicional Mediterránea. Barcelona: Grupo 4.

(7) Sorreche, M.A. (2004). Poblamiento y Arquitectura Tradicional en Granada, p. 136-149, Granada: COAATG.

(8) Feduchi, L. (1976/79). Itinerarios Arquitectura Popular Española. Barcelona: Blume.

(9) Flores, C. (1973). Arquitectura Popular Española. Tomo 4, Madrid: Aguilar.

(10) Blakstad, R. (2013). La Casa Eivissenca, Claus d'una tradició mil-lenaria, Ibiza: JJ Olañeta.

(11) Serra Rodríguez, J.J. (2000). Fortificacions Rurals a I’Illa d'Eivissa, Ibiza: Mediterrania.

(12) García Grinda, J.L. (2007). L'architecture traditionnelle méditerranéennee. Territoire, paysage et architecture traditionelle. En RehabiMed, Levaillant, M. (Trad.), Métode RehabiMed. Architecture Traditionnelle Méditerranéenne. II. Réhabilitation Bâtiments, (pp. 49-66), Barcelona: CAIAT.

(13) Mas Hernández, A. (1987). Arquitectura Popular del Campo de Cartagena. En Caro Baroja, J., Cea Gutierrez, A., Fernández Montes, M., Sánchez Gómez, L.A., Arquitectura Popular en España, (pp. 541-550) Madrid: CSIC.

(14) Muhler, A. (1982). La Arquitectura Rural de Ibiza como forma de Construcción Aglutinada. En Caro Baroja, J. (Ed), Arquitectura y Espacio Rural en Ibiza, Ibiza: COAIB.

(15) López Osorio, J.M., Abadía Molina, J. (1998). Cuevas, cubiertas y muros de tierra en la provincia de Granada. Encuentro Internacional Centro de Investigación Navapalos. Arquitectura de Tierra, (pp. 267-279). Madrid: Ministerio de Fomento.

(16) Guibbert, J.J. (1982). The Ecology and Ideology of Cities on the Edge of de Desert. En Reading The Contemporany African City, (pp. 9-26). Senegal: The Aga Khan Award for Architecture.

(17) Soriano Alfaro, V. (2006). Arquitectura de tierra en el sur de Marruecos: el oasis de Skoura. Barcelona: Fundación Caja de Arquitectos.

(18) Martín Arnaiz, M. (1976). Infiltración. En Custodio Gimena, E; Llamas Madurga, M.R. (Ed) Hidrología Subterránea (Capítulo 6.6.) Barcelona: Omega, S.A. 